JOURNAL OF

SYMPLECTIC GEOMETRY

Volume 7, Number 3, 337-355, 2009

\title{
RESOLUTION OF SYMPLECTIC CYCLIC ORBIFOLD SINGULARITIES
}

\author{
Klaus Niederkrüger and Federica Pasquotto
}

In this paper we present a method to obtain resolutions of symplectic orbifolds arising from symplectic reduction of a Hamiltonian $\mathbb{S}^{1}$-manifold at a regular value.

As an application, we show that all isolated cyclic singularities of a symplectic orbifold admit a resolution and that pre-quantizations of symplectic orbifolds are symplectically fillable by a smooth manifold.

\section{Introduction}

Symplectic quotients are an important source of new symplectic manifolds: they appear as symplectic reductions in the context of Hamiltonian actions and the associated moment maps. More generally, reduced spaces corresponding to regular values of the moment map turn out to be symplectic orbifolds, but one can still look for a closed smooth symplectic manifold which is isomorphic to the orbifold outside a neighbourhood of the singular set: we call this object a symplectic orbifold resolution.

Even in the case of a reduced space corresponding to a singular value of the moment map, where singularities of a more complicated type can occur, Kirwan's "partial desingularization" method [Kir85] can be applied to obtain a resolution which has only orbifold singularities.

The method described in this paper relies on the construction of an auxiliary circle action in a neighbourhood of the singularities with largest structure group: this action is subsequently used to perform a symplectic cut (as described in [Ler95]), which also amounts to a weighted blow up along the singular stratum [God01] as explained in Remark 3.5. The orbifold obtained in this way has singularities of lower order, and we can repeat the step inductively until we produce a smooth manifold. Making use of this procedure, we are able to find orbifold resolutions for reduced spaces obtained from symplectic reduction at the regular level sets of a Hamiltonian function generating an $\mathbb{S}^{1}$-action. In particular, we find symplectic resolutions 
for all isolated cyclic orbifold singularities. A technique involving symplectic resolutions of orbifolds via blow-ups can also be found in [CFM08]: due to a very restrictive definition of orbifolds, though, that result only provides resolutions in the special case of isolated singularities.

As a direct application of our result, we are able to show that a Seifert manifold with a contact structure that is $\mathbb{S}^{1}$-invariant and transverse to the fibres is symplectically fillable by a smooth manifold.

1.1. Isolated singularities in dimension four. We start our paper by discussing singularities of the simplest form: we hope that this will provide the reader with some motivation and will serve as the right introduction to the difficulties arising when considering more general examples.

In the four-dimensional case, we can give a very explicit description of the resolution of an isolated orbifold singularity. In order to do so, we use weighted blow-ups of isolated symplectic orbifold singularities, as defined in [God01].

Let $x \in\left(M^{(4)}, \omega\right)$ be an isolated orbifold singularity with structure group $\Gamma \cong \mathbb{Z}_{p}$. The orbifold chart around $x$ is equivariantly symplectomorphic to a neighbourhood of 0 in $\mathbb{C}^{2}$ with $\mathbb{Z}_{p}=\left\{1, \xi, \xi^{2}, \ldots, \xi^{p-1}\right\}$ acting by

$$
\left(z_{1}, z_{2}\right) \longmapsto\left(\xi^{m} z_{1}, \xi z_{2}\right), \quad 0<m<p, \quad \operatorname{gcd}(m, p)=1 .
$$

If $m$ and $p$ were not coprime, the orbifold singularity would not be isolated. We can define an $\mathbb{S}^{1}$-action on $\mathbb{C}^{2}$ by setting $\lambda \cdot\left(z_{1}, z_{2}\right)=\left(\lambda^{m} z_{1}, \lambda z_{2}\right)$ for $\lambda \in \mathbb{S}^{1}$. The actions of $\mathbb{Z}_{p}$ and $\mathbb{S}^{1}$ commute, but the induced circle action on $\mathbb{C}^{2} / \mathbb{Z}_{p}$ is not effective. Instead we have to go to the $\mathbb{S}^{1} / \mathbb{Z}_{p}$-action obtained from the following exact sequence:

$$
0 \longrightarrow \mathbb{Z}_{p} \longrightarrow \mathbb{S}^{1} \longrightarrow \hat{\mathbb{S}}^{1} \longrightarrow 0,
$$

with the homomorphism of the circle given by $\lambda \mapsto \lambda^{p}$. This defines now a symplectic $\hat{\mathbb{S}}^{1}$-action on $\mathbb{C}^{2} / \mathbb{Z}_{p}$ by

$$
\mu \cdot\left[z_{1}, z_{2}\right]=\left[\lambda \cdot\left(z_{1}, z_{2}\right)\right]=\left[\lambda^{m} z_{1}, \lambda z_{2}\right]
$$

for $\mu \in \hat{\mathbb{S}}^{1}$ and a $\lambda \in \mathbb{S}^{1}$ such that $\lambda^{p}=\mu$.

The weighted blow-up of $\mathbb{C}^{2} / \mathbb{Z}_{p}$ at the origin can be represented as a symplectic cut with respect to this $\hat{\mathbb{S}}^{1}$-action: Take the product orbifold $\mathbb{C}^{2} / \mathbb{Z}_{p} \times \mathbb{C}$ with the symplectic form given by $(\omega,-i d w \wedge d \bar{w})$ and the effective $\hat{\mathbb{S}}^{1}$-action

$$
\mu \cdot\left(\left[z_{1}, z_{2}\right], w\right)=\left(\mu \cdot\left[z_{1}, z_{2}\right], \mu^{-1} w\right)=\left(\left[\lambda^{m} z_{1}, \lambda z_{2}\right], \lambda^{-p} w\right), \quad \lambda^{p}=\mu .
$$

The blow-up is the symplectic reduction of this space. The Hamiltonian function that corresponds to this action is

$$
H\left(\left[z_{1}, z_{2}\right], w\right)=m\left|z_{1}\right|^{2}+\left|z_{2}\right|^{2}-p|w|^{2},
$$


and the $\varepsilon$-level set $H^{-1}(\varepsilon)$ is diffeomorphic to the manifold $\mathbb{S}^{3} / \mathbb{Z}_{p} \times \mathbb{C}$. The last step consists in taking the quotient $H^{-1}(\varepsilon) / \hat{\mathbb{S}}^{1}$ to obtain a symplectic orbifold that can be glued to $\mathbb{C}^{2} / \mathbb{Z}_{p}$ after removing a neighbourhood of the origin.

There is only one singular point in $H^{-1}(\varepsilon) / \hat{\mathbb{S}}^{1}$, namely the image of $([1,0], 0) \in \mathbb{S}^{3} / \mathbb{Z}_{p} \times \mathbb{C}$, with stabilizer $\mathbb{Z}_{m}$. By the slice theorem, a neighbourhood of this point admits an orbifold chart equivalent to $\mathbb{C}^{2}$ with $\mathbb{Z}_{m}$ acting by $\eta \cdot\left(w_{1}, w_{2}\right)=\left(\eta^{-p} w_{1}, \eta w_{2}\right)$.

Now choose $a_{1} \in \mathbb{Z}$ such that $0<a_{1} m-p<m$ and set $m_{1}=a_{1} m-p$ and $p_{1}=m$ : then the new singularity can also be modelled by $\mathbb{Z}_{p_{1}}$ acting by $\eta \cdot\left(z_{1}, z_{2}\right)=\left(\eta^{m_{1}} z_{1}, \eta z_{2}\right)$ with $\operatorname{gcd}\left(m_{1}, p_{1}\right)=1$, because if $b$ divides both $p_{1}$ and $m_{1}$ then it also divides $m$ and $p$. We are thus in the initial type of situation, but we have managed to reduce the order of the singularity. We can iterate this process, and progressively decrease the order of the singularity until we obtain, after a finite number of steps, a resolution of our initial orbifold chart.

More precisely, if we blow up a second time, we obtain a singularity with structure group $\mathbb{Z}_{m_{1}}$ acting by $\zeta \cdot\left(z_{1}, z_{2}\right)=\left(\zeta^{a_{2} m_{1}-p_{1}} z_{1}, \zeta z_{2}\right)$. If we iterate this blow-up process, at each step we replace the previous singularity by a new one with structure group $\mathbb{Z}_{p_{i}}$ acting by $\left(\xi^{m_{i}} z_{1}, \xi z_{2}\right)$, where the pair $\left(p_{i}, m_{i}\right)$ is recursively given by

$$
\left(\begin{array}{c}
p_{i} \\
m_{i}
\end{array}\right)=\left(\begin{array}{cc}
0 & 1 \\
-1 & a_{i}
\end{array}\right)\left(\begin{array}{c}
p_{i-1} \\
m_{i-1}
\end{array}\right)
$$

with each $a_{i}$ corresponding to the "roundup" of $\frac{p_{i-1}}{m_{i-1}}$, that is, the least integer $\geq \frac{p_{i-1}}{m_{i-1}}$. The sequence $\left[a_{1}, a_{2}, \ldots\right]$ corresponds to the continued fraction of $\frac{p}{m}$ (a description of resolutions in terms of continued fractions is contained, for example, in Miles Reid's lecture notes [Rei]). After sufficiently many blow-ups, we get a pair of the form $\left(p_{N}, 1\right)$ and thus an orbifold chart which is in fact smooth.

Notice that we can think of each weighted blow-up as taking the connected sum with a suitable orbifold. In dimension 4 , if we define the weighted projective space to be the quotient

$$
\mathbb{C P}\left(a_{0}, a_{1}, a_{2}\right)=\mathbb{C}^{3}-\{0\} / \sim
$$

under the equivalence relation

$$
\left(z_{0}: z_{1}: z_{2}\right) \sim\left(\lambda^{a_{0}} z_{0}: \lambda^{a_{1}} z_{1}: \lambda^{a_{2}} z_{2}\right) \text { for } \lambda \in \mathbb{C}^{*},
$$

then the weighted blow-up of a singular point with structure group $\mathbb{Z}_{p}$ acting by $\left(z_{1}, z_{2}\right) \mapsto\left(\xi^{a} z_{1}, \xi^{b} z_{2}\right)$ can be described as taking the connected sum, around this point, with the orbifold $\mathbb{C P}(a, b, p)$ with reversed orientation. We can use this description to represent the resolution of a four-dimensional cyclic singularity as in Figure 1. 


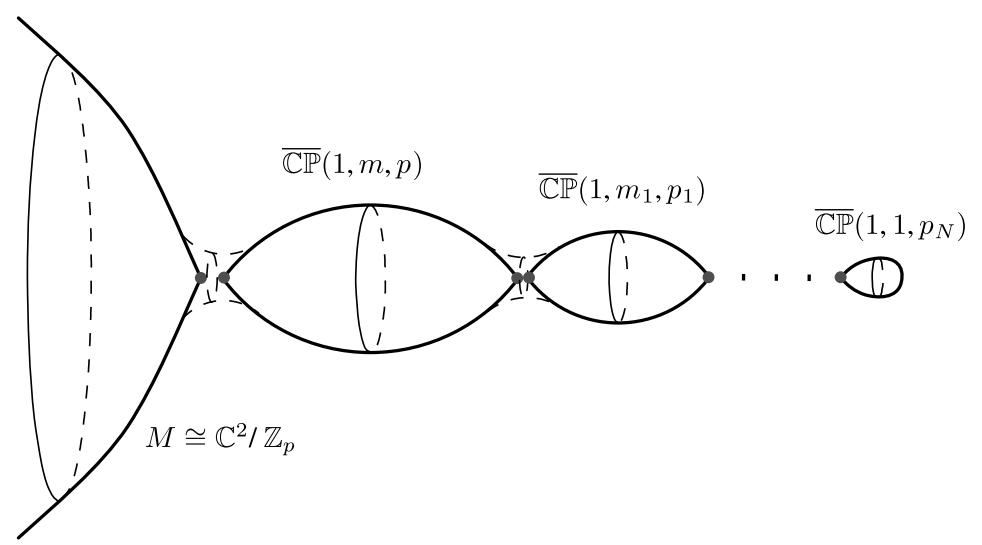

Figure 1. The resolution obtained via a sequence of blow-up can also be thought of as the connected sum $M \# \overline{\mathbb{C P}}(1, m, p) \# \overline{\mathbb{C P}}\left(1, m_{1}, p_{1}\right) \# \cdots \# \overline{\mathbb{C P}}\left(1,1, p_{N}\right)$.

In higher dimension $(\geq 6)$, the method just described is not so straightforward: even if we start with an isolated singularity, after the first blow-up the singular set is not necessarily discrete any more.

Example 1.1. Consider an isolated orbifold singularity modelled on a neighbourhood of 0 in $\mathbb{C}^{3}$ with $\mathbb{Z}_{p}$ acting by

$$
\left(z_{1}, z_{2}, z_{3}\right) \mapsto\left(\xi^{m_{1}} z_{1}, \xi^{m_{2}} z_{2}, \xi z_{3}\right)
$$

with $0<m_{1}<m_{2}<p$ and $\operatorname{gcd}\left(m_{j}, p\right)=1, j=1,2$. We can blow up this singularity with the method used in the four-dimensional case, that is, performing a symplectic cut with respect to a suitable circle action. If $\operatorname{gcd}\left(m_{1}, m_{2}\right)=d \neq 1$, though, after blowing up the singular set will be a two-dimensional suborbifold with stabiliser $\mathbb{Z}_{d}$ at generic points.

One could still hope to obtain a resolution of general cyclic orbifold singularities by using weighted blow-ups along suborbifolds (cf. [MS99]), but for this one needs to find a suitable circle action on the fibres of the normal orbibundle to singular strata. While this is not difficult in charts, it is not clear to us how to find in general a global action. To sketch the type of problems we encounter, consider the bundle $\mathbb{R}^{2} \times\left(\mathbb{C}^{2} / \mathbb{Z}_{5}\right) / \sim$ with $\left(t, s,\left[z_{1}, z_{2}\right]\right) \sim\left(t+1, s,\left[z_{2}, z_{1}\right]\right)$ and $\mathbb{Z}_{5}$ acting by $\xi\left(z_{1}, z_{2}\right)=\left(\xi z_{1}, \xi^{4} z_{2}\right)$. If we tried to proceed as in the case of isolated singularities, we would choose the circle action given by $\lambda\left[z_{1}, z_{2}\right]=\left[\lambda z_{1}, \lambda^{4} z_{2}\right]$, but the relation " $\sim$ " is not equivariant with respect to this action. In the special context of symplectic reduction on Hamiltonian $\mathbb{S}^{1}$-manifolds, we adopt a different desingularisation method. In fact, this also induces a circle action with the required 
properties on the quotient, so that our construction ultimately amounts to a weighted blow-up at the orbifold level (cf. the remark at the end of Section 3).

\section{Symplectic orbifolds as quotients of Hamiltonian $\mathbb{S}^{1}$-manifolds}

We start by recalling some definitions.

Definition. A symplectic orbifold $M$ is a Hausdorff, second countable topological space, equipped with an atlas of uniformizing charts $\left(\widetilde{U}_{i}, \Gamma_{i}, \varphi_{i}, \omega_{i}\right)$, where $\widetilde{U}_{i}$ is an open connected subset of $\mathbb{R}^{n}, \Gamma_{i}$ is a finite group of symplectomorphisms of $\left(\widetilde{U}_{i}, \omega_{i}\right)$ and $\varphi_{i}: \widetilde{U}_{i} \rightarrow M$ induces a homeomorphism from $\widetilde{U}_{i} / \Gamma_{i}$ to $U_{i} \subset M$. These charts are required to cover $M$ and to satisfy the following compatibility condition: if $x \in \widetilde{U}_{i}$ and $y \in \widetilde{U}_{j}$ are such that $\varphi_{i}(x)=\varphi_{j}(y)$ then there exists a symplectomorphism from a neighbourhood of $x$ onto a neighbourhood of $y$ whose composition with $\varphi_{j}$ is $\varphi_{i}$.

Let $W$ be a manifold with an action of the unit circle $\mathbb{S}^{1}$. The infinitesimal action is defined by the vector field

$$
X_{W}(p):=\left.\frac{d}{d t}\right|_{t=0} e^{i t} * p
$$

for every $p \in W$.

Definition. A Hamiltonian $\mathbb{S}^{1}$-manifold $(W, \omega)$ is a symplectic manifold with an $\mathbb{S}^{1}$-action for which there exists a Hamiltonian function $H: W \rightarrow \mathbb{R}$ that generates the action or, equivalently, that satisfies the identity

$$
i_{X_{W}} \omega=d H \text {. }
$$

If this is the case, the function $H$ is also called the moment map of the action.

The following statement is well known [Wei77], but for completeness we will briefly sketch the proof.

Proposition 2.1. Let $(W, \omega)$ be a Hamiltonian $\mathbb{S}^{1}$-manifold with Hamiltonian function $H$. For any regular value $E$ of $H$, the symplectic quotient

$$
M_{E}:=H^{-1}(E) / \mathbb{S}^{1}
$$

is a symplectic orbifold.

Proof. Since $E$ is a regular value, it follows that $H^{-1}(E)$ is a smooth submanifold on which the $\mathbb{S}^{1}$-action is locally free, because $i_{X_{W}} \omega=d H \neq 0$. The stabilizer of a point $p \in H^{-1}(E)$ is thus isomorphic to some finite cyclic subgroup. By the slice theorem, we can find a homeomorphism from a neighbourhood of the equivalence class of $p$ in the orbit space to the quotient $S_{p} / \operatorname{Stab}(p)$, where $S_{p}$ denotes a slice at $p$ (the normal space to the 
$\mathbb{S}^{1}$-orbit). In this way we obtain an orbifold atlas for the space $M_{E}$. Moreover, the symplectic form $\omega$ induces a symplectic structure on the orbifold $M_{E}$, because $\mathcal{L}_{X_{W}} \omega \equiv 0$, and $\left.\omega\left(X_{W}, \cdot\right)\right|_{T H^{-1}(E)}=\left.d H\right|_{T H^{-1}(E)} \equiv 0$.

Example 2.2. Let $\mathbb{C}^{n+1}$ be equipped with the canonical symplectic form $\omega_{0}=\frac{i}{2} \sum d z_{j} \wedge d \bar{z}_{j}$. The Hamiltonian function $H\left(z_{0}, \ldots, z_{n}\right)=$ $-\frac{1}{2} \sum a_{j}\left|z_{j}\right|^{2}$ generates the $\mathbb{S}^{1}$-action

$$
\lambda *\left(z_{0}, z_{1}, \ldots, z_{n}\right)=\left(\lambda^{a_{0}} z_{0}, \ldots, \lambda^{a_{n}} z_{n}\right) .
$$

The weighted projective space $\mathbb{C P}\left(a_{0}, a_{1}, \ldots, a_{n}\right)$ is the $2 n$-dimensional orbifold obtained as the symplectic reduction of $\mathbb{C}^{n+1}$ at any negative level set of $H$ (the choice of the level set only changes the scaling of the symplectic form).

\subsection{Symplectic resolutions}

Definition. Let $(M, \omega)$ be a symplectic orbifold with singular set $X$ and let $U$ be a neighbourhood of $X$. A symplectic resolution of $M$ on $U$ consists of a smooth symplectic manifold $(\widetilde{M}, \widetilde{\omega})$ and a continuous surjective map $p:(\widetilde{M}, \widetilde{\omega}) \rightarrow(M, \omega)$ which is a diffeomorphism on the complement of the singular set and a symplectomorphism outside $U$.

In this paper we prove the following result.

Theorem 2.3. Let $M$ be a symplectic orbifold arising from symplectic reduction of a Hamiltonian $\mathbb{S}^{1}$-manifold at a compact regular level set and let $X$ be the subset of orbifold singularities of $M$. Then for any neighbourhood $U$ of $X$ there exists a symplectic resolution of $M$ on $U$.

2.2. The stratification of the singular set. Let $W$ be a symplectic $\mathbb{S}^{1}$ manifold with Hamiltonian function $H$ and denote by Sing $W \subset W$ the singular set of the circle action, that is,

$$
\text { Sing } W=\{x \in W \mid \operatorname{Stab}(x) \neq\{1\}\} .
$$

For a given cyclic group $\mathbb{Z}_{k}$, let $W_{k}$ denote the union of orbits whose isotropy group is $\mathbb{Z}_{k}$, namely $W_{k}=\left\{x \in W \mid \operatorname{Stab}(x) \cong \mathbb{Z}_{k}\right\}$. Then Sing $W$ is stratified by singular strata, i.e., connected components of $W_{k}$ for all $k \neq 1$. Assume that 0 is a regular value of $H$ and denote by $P$ the level set $H^{-1}(0)$. We assume $P$ to be compact: this is always the case, for example, if the Hamiltonian function is proper. The singular set of the action on this level set is given by the intersection of Sing $W$ and $P$ : we will denote it by $\operatorname{Sing} P$. If $\pi: P \rightarrow M=P / \mathbb{S}^{1}$ denotes the orbit map, $X=\pi(\operatorname{Sing} P)$ is the set of orbifold singularities of $M$ and the stratification of $\operatorname{Sing} P$ descends to a stratification of $X$. Our desingularization method works by induction on the order of the stabilizers of the strata $W_{k}$ that have non-empty intersection with $P$. Namely, we start with the stratum with largest stabilizer (the 
minimal stratum, which exists because $\operatorname{Sing} P$ is compact), remove a small neighbourhood of it and glue in a (suitable) smooth manifold, in such a way that both the symplectic form and the Hamiltonian $\mathbb{S}^{1}$-action extend to the manifold resulting from this surgery. The new singular set will also carry a stratification, but the order of the stabilizer of the minimal stratum will be strictly less than $k$. If we successively repeat this procedure sufficiently often, we will eventually reduce this maximal stabiliser to the trivial group. In particular, symplectic reduction at 0 yields then a smooth symplectic manifold which gives a resolution of the orbifold $M$.

After working out this desingularization method for symplectic orbifolds, we were told by one of the authors of [GGK99] that they, and others before them, had already used the same construction in the smooth category.

\section{Construction of the resolution}

The strategy of our construction is to find an auxiliary circle action on the Hamiltonian $\mathbb{S}^{1}$-manifold around its minimal stratum. This action will have certain properties (see Proposition 3.3) that will allow us to perform a symplectic cut [Ler95]. The stabilizers of the Hamiltonian $\mathbb{S}^{1}$-manifold obtained this way are all lower than the stabilizer of the initial minimal stratum. Then we successively repeat the construction until we obtain a manifold with a free circle action.

3.1. An auxiliary circle action around the minimal stratum. Suppose $(W, \omega)$ is a Hamiltonian $\mathbb{S}^{1}$-manifold with Hamiltonian function $H$ : $W \rightarrow \mathbb{R}$. Assume further that 0 is a regular level set of $H$ and that the singular set of the action on $P:=H^{-1}(0)$ is compact. Choose a metric and an almost complex structure $J$ which are $\mathbb{S}^{1}$-invariant and compatible with $\omega$ (see for example [MS98, Section 5.5]). Since symplectic reduction is a local process, it will be sufficient to study a neighbourhood of $P$ consisting of regular level sets $P_{t}:=H^{-1}(t), t \in(-\varepsilon, \varepsilon)$. We will start by finding a suitable model for this neighbourhood.

Proposition 3.1. There is a neighbourhood of $P$ in $(W, \omega)$ that is $\mathbb{S}^{1}$ diffeomorphic to

$$
P \times(-\varepsilon, \varepsilon),
$$

with trivial circle action on the second factor $\lambda *(p, t)=(\lambda * p, t)$. Under this diffeomorphism, the Hamiltonian function pulls back to $H(p, t)=t$.

Proof. Consider the vector field

$$
Y:=\frac{1}{\|\nabla H\|^{2}} \nabla H=\frac{1}{\left\|X_{W}\right\|^{2}} J X_{W} .
$$


It is transverse to the level sets, and allows us to define for small times $t$ with $|t|<\varepsilon$ the diffeomorphism

$$
\Psi: P \times(-\varepsilon, \varepsilon) \rightarrow W,(p, t) \mapsto \Phi_{t}^{Y}(p)
$$

onto a neighbourhood of $P$ in $W$. The pullback of $H$ gives $H \circ \Psi(p, t)=t$, and the circle action is $t$-invariant (by which we mean $\lambda * \Psi(p, t)=\Psi(\lambda * p, t)$ for $\left.\lambda \in \mathbb{S}^{1}\right)$, because $Y$ commutes with the generator of the $\mathbb{S}^{1}$-action.

From now on, we will restrict to a neighbourhood of the level set $P$ as constructed in Proposition 3.1 and for simplicity we will refer to it as $W$.

In the next step, we will construct a suitable model for a neighbourhood of the minimal stratum, in which we can describe the auxiliary circle action needed for the resolution.

The $\mathbb{S}^{1}$-action on $W$ is locally free. Consider the stratification $\left\{W_{k}\right\}$ of the singular set $\operatorname{Sing} W$ given by the isotropy groups. Each stratum $W_{k}$ is an $\mathbb{S}^{1}$-invariant symplectic submanifold of $W$ of the form $P_{k} \times(-\varepsilon, \varepsilon)$, where $P_{k}:=W_{k} \cap P$.

If $k$ is maximal, that is, there are no points in $W$ of order larger than $k$, then $W_{k} \cap P_{t}$ is a closed submanifold. By restricting to one component, we may further assume $W_{k}$ to be connected. Choose any $\mathbb{S}^{1}$-invariant metric $g_{P}$ on $P$ and extend it to the product metric $g_{W}:=g_{P} \oplus d t^{2}$ on $W=P \times(-\varepsilon, \varepsilon)$. With our choice of metrics on $P$ and $W$, the corresponding exponential maps are related as follows:

$$
\exp _{(p, t)}^{W}\left(v+a \partial_{t}\right)=\left(\exp _{p}^{P}(v), t+a\right)
$$

with respect to the splitting $T_{(p, t)} W=T_{p} P \oplus\left\langle\partial_{t}\right\rangle$. Moreover, if we denote the normal bundle of $P_{k}$ in $P$ by $N_{k}$, then the normal bundle of $W_{k}$ in $W$ is isomorphic to the product $N_{k} \times(-\varepsilon, \varepsilon)$. Therefore a tubular neighbourhood of $W_{k}$ in $W$ can also be identified via the exponential map with the product $N_{k}(\delta) \times(-\varepsilon, \varepsilon)$, where $N_{k}(\delta)$ denotes the $\delta$-subdiskbundle of $N_{k}$. This can be summarized in the following proposition:

Proposition 3.2. There exists a neighbourhood of the singular stratum $W_{k}$ in $W$ that has the form $N_{k}(\delta) \times(-\varepsilon, \varepsilon)$. The Hamiltonian function on this neighbourhood is just given by $H(v, t)=t$ and the circle action is the linearized $\mathbb{S}^{1}$-action on $N_{k}$.

In what follows, we will therefore implicitly assume this identification and denote for simplicity by $\omega$ and $J$ the pullback of the corresponding structures on $W$.

Later it will be necessary to introduce a second circle action. To avoid confusions, from now on we will call the linearization of the given action the $\beta$-action, and we will write it as $\lambda *_{\beta} v$ for $\lambda \in \mathbb{S}^{1}$ and $v \in N_{k}(\delta)$.

Let $x \in W_{k}$ be a singular point in the minimal stratum. The stabilizer $\operatorname{Stab}(x) \cong \mathbb{Z}_{k}$ acts by isometric (with respect to both $g_{W}$ and 
the metric $\left.g_{J}:=\omega(\cdot, J \cdot)\right)$, J-linear transformations on $T_{x} W$, hence there exists an isomorphism between the Hermitian vector spaces $\left(T_{x} W, J, g_{J}\right)$ and the standard Hermitian space $\mathbb{C}^{n}$ such that the linearized $\mathbb{Z}_{k}$-action takes the form

$$
\lambda *_{\beta} \mathbf{z}=\left(\lambda^{\widetilde{a}_{1}} z_{1}, \ldots, \lambda^{\widetilde{a}_{n}} z_{n}\right),
$$

for $\lambda \in \mathbb{Z}_{k}, \mathbf{z} \in \mathbb{C}^{n} \cong T_{x} W$, and some $\widetilde{a}_{1}, \ldots, \widetilde{a}_{n} \in \mathbb{Z}$. Without loss of generality we may assume that $0=\widetilde{a}_{1}=\cdots=\widetilde{a}_{m}<\widetilde{a}_{m+1} \leq \cdots \leq \widetilde{a}_{n}<k$ for some $m$. The first $m$ directions span the space $T_{x} W_{k}$, and the other directions are orthogonal to $T_{x} W_{k}$ : it is easy to show that this holds not only for $g_{J}$, but also with respect to any other $\mathbb{S}^{1}$-invariant metric. Hence, if we denote by $\nu_{k}$ the normal bundle of $W_{k}$ in $W$, the subspace $\left\{\left(0, \ldots, 0, z_{m+1}, \ldots, z_{n}\right)\right\}$ coincides with the fibre $\nu_{k}(x)$. For all $x \in W_{k}, j_{x}:=\left.J\right|_{\nu_{k}(x)}$ defines a complex vector bundle structure on $\nu_{k}$, making the $\mathbb{Z}_{k}$-action $j$-complex linear in each fibre.

Denote by $a_{1}<\cdots<a_{l}$ the distinct exponents occurring in the normal form for the action: $\nu_{k}$ splits thus into a direct sum of subbundles

$$
\nu_{k}=E_{1} \oplus \cdots \oplus E_{l},
$$

where $E_{i}(x)$ denotes the eigenspace corresponding to the eigenvalue $\lambda^{a_{i}}$ in the fibre at the point $x$. This splitting is well defined for each component of $W_{k}$. Therefore we can extend the $\mathbb{Z}_{k}$-action to a second circle action by setting for any $\lambda \in \mathbb{S}^{1}$

$$
\lambda *_{\text {aux }} v:=\lambda^{a_{1}} v_{1}+\cdots+\lambda^{a_{l}} v_{l},
$$

where $v=v_{1}+\cdots+v_{l}$ is a splitting with respect to the eigenspaces defined above, and $\lambda^{a_{i}} v_{i}:=\left(\cos \left(a_{i} \lambda\right)+\sin \left(a_{i} \lambda\right) j\right) v_{i}$. This auxiliary action is fibrewise and $j$-linear, and commutes with the original $\beta$-action. Unfortunately, it does not need to respect the symplectic form $\omega$. Recall that $\omega$ denotes here the symplectic form on the total space of $\nu_{k}$ obtained by pulling back the symplectic form on $W$. By averaging $\omega$ over the auxiliary action, we obtain a closed 2-form $\omega^{\prime}$ on $\nu_{k}$ that is invariant with respect to both the $\beta$ - and the auxiliary action. There is a small neighbourhood of the zero section of $\nu_{k}$ where we also have $\left(\omega^{\prime}\right)^{n} \neq 0$ and hence $\omega^{\prime}$ is a symplectic form. To prove this it suffices to show that $\omega$ is aux-invariant on the zero section. In fact, at the zero section $W_{k}$ of $\nu_{k}$, there is a well-defined splitting of the tangent bundle $\left.T \nu_{k}\right|_{W_{k}}=T W_{k} \oplus \nu_{k}$ and we can write

$$
\left.T \nu_{k}\right|_{W_{k}}=T W_{k} \oplus E_{1} \oplus \cdots \oplus E_{l} .
$$

The $E_{i}$ 's are $J$-linear subspaces and $g_{J}$-orthogonal to each other, so that they are also symplectically orthogonal. The linearized auxiliary action on a vector $w+v_{1}+\cdots+v_{l} \in T \nu_{k}$ with $w \in T_{x} W_{k}$ and $v_{i} \in E_{i}(x)$ is given by

$$
\lambda *_{\text {aux }}\left(w+v_{1}+\cdots+v_{l}\right)=w+\lambda^{a_{1}} v_{1}+\cdots+\lambda^{a_{l}} v_{l}
$$


and using the orthogonality relations, it is easy to check that

$$
\omega\left(\lambda *_{\text {aux }} v, \lambda *_{\text {aux }} v^{\prime}\right)=\omega\left(v, v^{\prime}\right) .
$$

Hence $\omega$ is aux-invariant on the zero section of $\nu_{k}$ and we do not change it there by averaging. It follows that there is a small neighbourhood of the zero section, where $\omega^{\prime}$ will be symplectic.

Proposition 3.3. There exists a Hamiltonian $\mathbb{S}^{1}$-action $\varphi$ in a neighbourhood of $W_{k}$ with the following properties:

(i) $\varphi$ leaves $W_{k}$ pointwise fixed and the stabilizer of points not lying on $W_{k}$ is a proper subgroup of $\mathbb{Z}_{k}$;

(ii) $\varphi$ commutes with the $\beta$-action;

(iii) the two actions coincide for elements in $\mathbb{Z}_{k} \leq \mathbb{S}^{1}$, and if $\lambda_{*_{\varphi}} x=\sigma *_{\beta} x$, then $\sigma \in \mathbb{Z}_{k} \leq \mathbb{S}^{1}$

(iv) the Hamiltonian function for the $\varphi$-action is $\beta$-invariant and constant along $W_{k}$. Furthermore, it is a Morse-Bott function with critical set $W_{k}$ of index zero.

Proof. We will obtain the $\varphi$-action by a deformation of the existing auxiliary action. By a standard application of the equivariant Moser trick, there exists a $\beta$-equivariant isotopy of a neighbourhood of $W_{k}$ that deforms $\omega$ into $\omega^{\prime}$. The only difficulty comes from the fact that we are dealing with an open set, so that the flow of the Moser vector field $X_{t}$ does not need to exist up to time 1 . But since $X_{t}$ vanishes on the zero section $W_{k}$, after shrinking $W_{k}$ and the radius of the tubular neighbourhood, we get a smaller set where the isotopy can be defined as the flow of $X_{t}$ for all $t \in[0,1]$. The inverse of this isotopy deforms the auxiliary action into the required action $\varphi$.

Since the flow of the Moser vector field leaves $W_{k}$ invariant and is $\beta$-equivariant, one can show that properties (i)-(iii) hold for the auxiliary action together with $\beta$ and this is equivalent to the corresponding statements for $\varphi$ and $\beta$.

Since $\omega$ is $\varphi$-invariant, one has that $d i_{X_{\varphi}} \omega=\mathcal{L}_{X_{\varphi}} \omega=0$. For the time being, let $U$ be any tubular neighbourhood of $W_{k}$, where $\varphi$ is defined. The closed 1-form $i_{X_{\varphi}} \omega$ represents a class in $H^{1}(U)$ which vanishes if we pull it back to the zero section $W_{k}$ : Given that $H^{1}(U) \cong H^{1}\left(W_{k}\right)$, it follows that $i_{X_{\varphi}} \omega$ is exact on $U$, i.e., there exists a function $\mu_{\varphi}$ such that $i_{X_{\varphi}} \omega=d \mu_{\varphi}$. The function $\mu_{\varphi}$ is uniquely defined up to an additive constant (which we may choose such that $\mu_{\varphi} \equiv 0$ on $W_{k}$ ) and is $\beta$-invariant.

Recall that a Hamiltonian $\mathbb{S}^{1}$-function is always Morse-Bott (see [MS98, Section 5.5]). The critical set coincides with the set of fixed points of the action, hence in our case with $W_{k}$.

The index of a Morse-Bott function is invariant under diffeomorphisms. Therefore in order to compute the index of $\mu_{\varphi}$ at $W_{k}$, it suffices to compute 
the index of the Hamiltonian function $\mu_{\mathrm{aux}}$ of the auxiliary action with respect to $\omega^{\prime}$, because $\mu_{\varphi}$ is the pullback of $\mu_{\text {aux }}$ under the Moser-flow.

Let $\partial_{r}$ be the radial vector field on $\nu_{k}$ given by

$$
\partial_{r}(v)=\left.\frac{d}{d t}\right|_{t=1} t \cdot v
$$

for $v \in \nu_{k}$. We will prove that $\mu_{\text {aux }}$ strictly increases in radial direction, which shows that it has index zero. More precisely, we will prove that $\mathcal{L}_{\partial_{r}} \mu_{\text {aux }} \geq 0$ with equality only at the zero section. By definition of $\mu_{\text {aux }}$, one has $i_{\partial_{r}} d \mu_{\text {aux }}=\omega^{\prime}\left(X_{\text {aux }}, \partial_{r}\right)$, so it will suffice to show that there exists a neighbourhood of $W_{k}$ where $\omega^{\prime}\left(X_{\text {aux }}, \partial_{r}\right) \geq 0$.

With $\pi$ denoting the bundle projection $\nu_{k} \rightarrow W_{k}$, the vertical bundle $V\left(\nu_{k}\right) \leq T \nu_{k}$ of $\nu_{k}$ can be identified with the pullback bundle

$$
\pi^{*} \nu_{k}=\left\{(v, w) \in \nu_{k} \times \nu_{k} \mid \pi(v)=\pi(w)\right\} .
$$

The identification of $\pi^{*}\left(\nu_{k}\right)$ and $V\left(\nu_{k}\right)$ goes as follows:

$$
\chi: \pi^{*}\left(\nu_{k}\right) \rightarrow V\left(\nu_{k}\right),\left.\quad(v, w) \mapsto \frac{d}{d t}\right|_{t=0}(v+t w) .
$$

Let $v \in \nu_{k}$, and write it as $v=v_{1}+\cdots+v_{l}$ with respect to the splitting $\nu_{k}=E_{1} \oplus \cdots \oplus E_{l}$. Then the vectors $X_{\text {aux }}$ and $\partial_{r}$ are given by

$$
X_{\text {aux }}(v)=\chi\left(v, a_{1} j v_{1}+\cdots+a_{l} j v_{l}\right) \quad \text { and } \quad \partial_{r}(v)=\chi(v, v)
$$

as elements of $V\left(\nu_{k}\right) \cong \pi^{*}\left(\nu_{k}\right)$. Now assume $v=(x, 0)$ lies in the zero section of $\nu_{k}$. Then

$$
\omega^{\prime}\left(\chi\left(v, \sum a_{i} j w_{i}\right), \chi\left(v, w_{1}+\cdots+w_{l}\right)\right)=\sum_{j=1}^{l} a_{j} \omega^{\prime}\left(j \chi\left(v, w_{j}\right), \chi\left(v, w_{j}\right)\right)>0
$$

if $w \neq 0$, since the eigenspaces $E_{j}$ 's are $\omega$-orthogonal. By continuity this also holds for all $v$ in a neighbourhood of the zero section and all $w \neq 0$. Hence in particular $\omega^{\prime}\left(X_{\mathrm{aux}}, \partial_{r}\right)>0$ on $U-W_{k}$.

3.2. Surgery along the minimal stratum. In this section we describe how to replace a neighbourhood of (one component of) the minimal stratum $W_{k}$ by a smooth manifold, and extend both the symplectic form $\omega$ and the Hamiltonian $\mathbb{S}^{1}$-action $\beta$ to the resulting manifold, in such a way that the singular points of the extended action are of order strictly smaller than $k$. In order to achieve this, we perform a symplectic cut with respect to the Hamiltonian $\mathbb{S}^{1}$-action $\varphi$ constructed in the previous section. The main reference for symplectic cuts and hence for everything that follows is Lerman's original paper [Ler95].

As in the previous section, consider the minimal singular stratum $W_{k}$, denote by $U$ a tubular neighbourhood in $W$, where the $\varphi$-action is defined, and take now the product $U \times \mathbb{C}$. It admits a first circle action, which is 
just the extension of the original $\mathbb{S}^{1}$-action $\beta$ by the trivial action on the $\mathbb{C}$-factor, namely, for $x \in U, z \in \mathbb{C}$

$$
\lambda *_{\beta}(x, z):=\left(\lambda *_{\beta} x, z\right),
$$

and we can define a second circle action on $U \times \mathbb{C}$ by setting

$$
\lambda *_{\varphi}(x, z)=\left(\lambda *_{\varphi} x, \lambda^{-k} z\right) .
$$

These two actions commute and therefore we can combine them and define a new $\mathbb{S}^{1}$-action

$$
\lambda *_{\tau}(x, z):=\lambda *_{\varphi}\left(\lambda^{-1} *_{\beta}(x, z)\right) .
$$

This $\tau$-action is not effective, because the $\varphi$ - and the $\beta$-action coincide for elements in $\mathbb{Z}_{k}$. Hence consider the short exact sequence

$$
0 \rightarrow \mathbb{Z}_{k} \rightarrow \mathbb{S}^{1} \rightarrow \hat{\mathbb{S}}^{1} \rightarrow 0
$$

with the homomorphism of the circle given by $\lambda \mapsto \lambda^{k}$, and let $\hat{\mathbb{S}}^{1}$ act on $U \times \mathbb{C}$ by $\sigma *_{\hat{\tau}}(x, z)=\lambda *_{\tau}(x, z)$ for some $\lambda \in \mathbb{S}^{1}$ such that $\lambda^{k}=\sigma$. This new action, which we denote by $\hat{\tau}$, is not only effective but by Proposition 3.3 (iii), even free and the quotient $(U \times \mathbb{C}) / \hat{\tau}$ is a smooth manifold. It still carries an $\mathbb{S}^{1}$-action induced by the $\varphi$-action on $U \times \mathbb{C}$, and this is well defined because $\varphi$ commutes with $\hat{\tau}$.

We define a symplectic form $\Omega=(\omega,-i d z \wedge d \bar{z})$ on $U \times \mathbb{C}$, which is invariant with respect to the $\hat{\tau}$-action. By construction, the infinitesimal generator of this action can be written as $X_{\hat{\tau}}=-X_{\beta}+X_{\varphi}$. The Hamiltonian for the $\hat{\tau}$-action is given by

$$
H_{\hat{\tau}}(x, z)=\mu_{\varphi}(x)-k|z|^{2}-H(x),
$$

and if we now do symplectic reduction at some level $\varepsilon$ we get the quotient $H_{\hat{\tau}}^{-1}(\varepsilon) / \hat{\tau}$ which, with the structure induced by $\Omega$ and $\varphi$, is a smooth Hamiltonian $\mathbb{S}^{1}$-manifold with Hamiltonian function $H_{\varphi}[(x, z)]=\mu_{\varphi}(x)-k|z|^{2}=$ $H(x)+\varepsilon$. Notice that $H_{\hat{\tau}}^{-1}(\varepsilon)$ can be written as the disjoint union of two $\hat{\tau}$-invariant manifolds

$$
\begin{aligned}
H_{\hat{\tau}}^{-1}(\varepsilon)= & \left\{\left.(x, z)\left|\mu_{\varphi}(x)-H(x)>\varepsilon,\right| z\right|^{2}=\frac{\mu_{\varphi}(x)-H(x)-\varepsilon}{k}\right\} \\
& \bigsqcup\left\{(x, 0) \mid \mu_{\varphi}(x)-H(x)=\varepsilon\right\} .
\end{aligned}
$$

Since the level sets of $\mu_{\varphi}$ and $H$ are transverse, the restriction of $\mu_{\varphi}$ to $P=H^{-1}(0)$ is still a Morse-Bott function of index zero. Hence we can choose $\delta>0$ such that $\mu_{\varphi}^{-1}(\delta) \cap P$ is contained in the interior of $U$ and has the structure of a sphere bundle over $P_{k}=P \cap W_{k}$. Choose $\varepsilon^{\prime}>0$ such that

$$
\left(\mu_{\varphi}-H\right)^{-1}(\delta / 2, \delta) \cap P_{t}
$$


is non-empty, does not intersect $W_{k}$, and is contained in the interior of $U$ for all $|t|<\varepsilon^{\prime}$ (see Figure 2). Assume from now on that all subsets in $W$ are restricted to $H^{-1}\left(\left(-\varepsilon^{\prime}, \varepsilon^{\prime}\right)\right)$. Then

$$
U(\delta / 2, \delta):=\left(\mu_{\varphi}-H\right)^{-1}(\delta / 2, \delta)
$$

is diffeomorphic to a spherical shell bundle over $W_{k}$. Choose now $\varepsilon=\delta / 2$, set $V:=H_{\hat{\tau}}^{-1}(\varepsilon) / \hat{\tau}$, and consider the map

$$
\Phi: U(\varepsilon, 2 \varepsilon) \rightarrow V, \quad x \mapsto\left[x, \sqrt{\frac{\mu_{\varphi}(x)-H(x)-\varepsilon}{k}}\right] .
$$

This is a diffeomorphism (onto its image), equivariant with respect to the $\beta$-action on $U(\varepsilon, 2 \varepsilon)$ and the $\varphi$-action on $V$. Its inverse can be constructed as follows: given $[x, z]$ with $z \neq 0$, we first represent the same class by an element $\left(x^{\prime}, z^{\prime}\right)$ such that $z^{\prime}$ is a real positive number, and then define

$$
\Phi^{-1}([x, z]):=x^{\prime} .
$$

Moreover, since $\Phi$ factors through a map $U(\varepsilon, 2 \varepsilon) \rightarrow H_{\hat{\tau}}^{-1}(\varepsilon)$ which is the identity in the first component and a real function in the second one, we have

$$
\Phi^{*}(\omega,-i d z \wedge d \bar{z})=\omega,
$$

hence $\Phi$ gives in fact an equivariant symplectic identification of $U(\varepsilon, 2 \varepsilon)$ with its image under $\Phi$. More precisely, we have

$$
\Phi(U(\varepsilon, 2 \varepsilon))=\left\{[x, z] \in V \mid \varepsilon<\mu_{\varphi}(x)-H(x)<2 \varepsilon\right\} .
$$

We can now remove a tubular $\varepsilon$-neighbourhood $\overline{U(\varepsilon)}$ of $W_{k}$ in $W$ and glue in the smooth manifold

$$
V(\varepsilon):=\left\{\left.(x, z)\left|\varepsilon \leq \mu_{\varphi}(x)-H(x)<2 \varepsilon,\right| z\right|^{2}=\frac{\mu_{\varphi}(x)-H(x)-\varepsilon}{k}\right\} / \hat{\tau}
$$

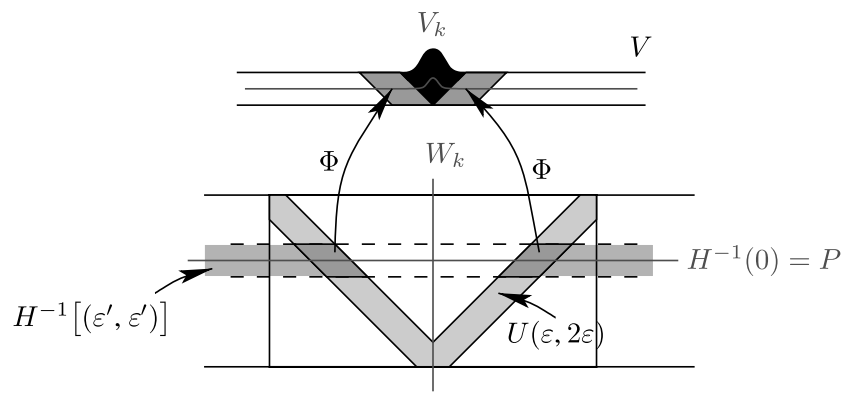

Figure 2. Remove the $U(\varepsilon)$-neighbourhood of $W_{k}$, and glue in the $V(\varepsilon)$-patch, identifying along $U(\varepsilon, 2 \varepsilon)$ via the diffeomorphism $\Phi$. 
along the open "collar" $U(\varepsilon, 2 \varepsilon)$, using the map $\Phi$. In this way we define the new manifold

$$
\widetilde{W}=(W-\overline{U(\varepsilon)}) \cup_{\Phi} V(\varepsilon) .
$$

Since $\Phi$ is equivariant, the $\beta$-action on $W-\overline{U(\varepsilon)}$ and the $\varphi$-action on $V(\varepsilon)$ fit together to give a circle action $\widetilde{\beta}$ on $\widetilde{W}$, which by construction coincides with $\beta$ outside a $2 \varepsilon$-neighbourhood of $W_{k}$. Moreover, $\Phi$ identifies the given symplectic forms on the two sides of the gluing, so $\widetilde{W}$ also admits a symplectic form $\widetilde{\omega}$ with the property that $\widetilde{\omega}=\omega$ on $W-U(2 \varepsilon)$. With the action $\widetilde{\beta}$ and the symplectic form $\widetilde{\omega}$ just defined, $\widetilde{W}$ is a Hamiltonian $\mathbb{S}^{1}$-manifold. The Hamiltonian function $\widetilde{H}$ for $\widetilde{\beta}$ is given by $H$ on $W-U(2 \varepsilon)$ and by $H_{\varphi}-\varepsilon$ on $V(\varepsilon)$.

We need to analyse the singular points of the $\widetilde{\beta}$-action on the "patch" $V(\varepsilon)$. They satisfy the relation $\lambda *_{\varphi}[x, z]=[x, z]$ for some $\lambda \in \mathbb{S}^{1}$ and this in turn means that there exist $\kappa \in \widehat{\mathbb{S}}^{1}$ and $\sigma \in \mathbb{S}^{1}, \sigma^{k}=\kappa$ such that

$$
\lambda *_{\varphi}(x, z)=\kappa *_{\hat{\tau}}(x, z)=\sigma *_{\varphi} \sigma^{-1} *_{\beta}(x, z) .
$$

In particular, $\lambda *_{\varphi} x=\sigma *_{\varphi} \sigma^{-1} *_{\beta} x$, and by Proposition 3.3 (iii) this identity can only hold if $\sigma \in \mathbb{Z}_{k}$. Hence $\kappa=1$ and singular points are characterized by $\lambda *_{\varphi}(x, z)=(x, z)$. Since $[x, z] \in V(\varepsilon)$ implies that $x$ does not lie in $W_{k}$, the isotropy groups are proper subgroups of $\mathbb{Z}_{k}$, see Proposition 3.3 (i).

We have thus proved:

Proposition 3.4. Let $W_{k}$ be minimal among the singular strata of a Hamiltonian $\mathbb{S}^{1}$-manifold $W$ that intersect the regular level set $P=H^{-1}(0)$. If we restrict to a suitable neighbourhood of $P$, we can remove an arbitrarily small neighbourhood of $W_{k}$ and symplectically glue in a smooth manifold which admits a Hamiltonian $\mathbb{S}^{1}$-action that coincides with the given action away from $W_{k}$ and only has singularities of order strictly smaller than $k$.

In fact, we have also shown that 0 is still a regular value of the moment map of the circle action on $\widetilde{W}$ and if we consider the symplectic reduced space at this level, namely

$$
\widetilde{M}:=\widetilde{H}^{-1}(0) / \widetilde{\beta},
$$

we see that this symplectic orbifold has singularities of strictly lower order than those of $M=H^{-1}(0) / \beta$.

Moreover, there exists a map $f: \widetilde{M} \rightarrow M$, which is a symplectic orbifold isomorphism outside an arbitrarily small neighbourhood of $M_{k}=P_{k} / \beta$ (and in fact coincides with the identity map outside a slightly larger neighbourhood). We shall describe how to define $f$. On $(P-U(2 \varepsilon)) / \beta$ it is simply the identity. In order to define it on $\left(V(\varepsilon) \cap H_{\varphi}^{-1}(\varepsilon)\right) / \varphi$ a little more work is required. First of all, denote by $V_{k}$ the quotient

$$
\left\{(x, 0) \in H_{\hat{\tau}}^{-1}(\varepsilon) \mid \mu_{\varphi}(x)-H(x)=\varepsilon\right\} / \hat{\tau} .
$$


Then the inverse of the gluing map $\Phi$ restricts to a diffeomorphism $\Phi^{-1}$ : $\left(V-V_{k}\right) \cap H_{\varphi}^{-1}(\varepsilon) \rightarrow P \cap U(\varepsilon, 2 \varepsilon)$. Since $\Phi$ is equivariant with respect to the $\varphi$ - and $\beta$-actions, this descends to a symplectic orbifold isomorphism on the quotients. Let $h: U(\varepsilon, 2 \varepsilon) \cap P \rightarrow\left(U(2 \varepsilon)-W_{k}\right) \cap P$ be a $\beta$ - and $\varphi$-equivariant diffeomorphism (recall that $\varphi$ is the action on $W$ constructed in Section 3.1), that is the identity in a neighbourhood of the outer boundary of $U(2 \varepsilon)$ and extends to a smooth map from $U(2 \varepsilon) \cap P$ to itself which maps $U(\varepsilon) \cap P$ to $P_{k}$.

Then we can define

$$
\begin{aligned}
& f: \quad\left(V \cap H_{\varphi}^{-1}(\varepsilon)\right) / \varphi \rightarrow(P \cap U(2 \varepsilon)) / \beta
\end{aligned}
$$

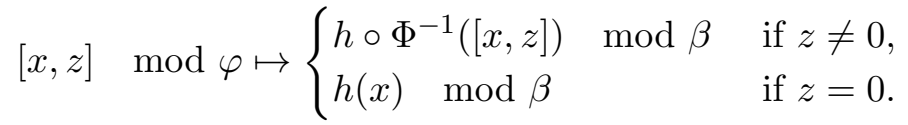

Because of the boundary conditions on $h$, the map $f$ extends on the outer side to the identity map. To see that $f$ is continuous in a neighbourhood of $\left(V_{k} \cap H_{\varphi}^{-1}(\varepsilon)\right) / \varphi$, one has to show that for any sequence $\left[x_{k}, z_{k}\right] / \varphi \subset$ $\left(V \cap H_{\varphi}^{-1}(\varepsilon)\right) / \varphi$ that converges to some element $\left[x_{0}, 0\right] / \varphi$, it follows that $f\left(\left[x_{k}, z_{k}\right] / \varphi\right)$ converges to $f\left(\left[x_{0}, 0\right] / \varphi\right)$. We can find representatives $\left(x_{k}^{\prime}, z_{k}^{\prime}\right)$ for the sequence that converge to $\left(x_{0}^{\prime}, 0\right)$ and such that $z_{k}^{\prime}$ is a non-negative real number, and hence $f\left(\left[x_{k}^{\prime}, z_{k}^{\prime}\right] / \varphi\right)=h\left(x_{k}^{\prime}\right) / \beta$ converges to $h\left(x_{0}^{\prime}\right) / \beta=$ $f\left(\left[x_{0}^{\prime}, 0\right] / \varphi\right)=f\left(\left[x_{0}, 0\right] / \varphi\right)$.

Remark 3.5. The above $\varphi$-action descends to a circle action defined on a neighbourhood of the minimal stratum in the symplectic quotient $M$. Our surgery construction induces on the orbifold level a weighted blow up along the minimal stratum with respect to this action [God01, Section 4].

Note that the symplectic weighted blow up does not define a unique construction, but depends on the choice of a local circle action. Only a suitable choice will reduce the order of the orbifold singularities, and ultimately yield a resolution. For general symplectic cyclic orbifolds (that are not given as symplectically reduced spaces), we have not been able to find an action that would allow us to produce a resolution by using weighted blow ups.

Our method amounts to replacing a neighbourhood of the minimal singular stratum in the orbifold with a patch of the form

$$
\left(\left\{H_{\hat{\tau}}=\varepsilon\right\} / \hat{\tau} \cap\left\{H_{\varphi}=\varepsilon\right\}\right) / \varphi,
$$

where $H_{\hat{\tau}}$ is the Hamiltonian function for the $\hat{\tau}$-action on $U \times \mathbb{C}$, and $H_{\varphi}$ is the Hamiltonian for the induced $\varphi$-action on $H_{\hat{\tau}}^{-1}(\varepsilon) / \hat{\tau}$.

The weighted blow up on the orbifold would replace the neighbourhood of the singular stratum in $M$ with a patch of the form

$$
\left(\left(\left\{H^{-1}(0)\right\} / \beta \times \mathbb{C}\right) \cap\left\{\hat{H}_{\varphi}=\varepsilon\right\}\right) / \varphi,
$$


where $H$ is the Hamiltonian for the $\beta$-action on $U$ and $\hat{H}_{\varphi}$ the Hamiltonian for the induced $\varphi$-action on a small open neighbourhood of the singularities of $\mathrm{M} \times \mathbb{C}$. It is easy to check by using commutativity of the actions and the relation between $H, H_{\varphi}$ and $H_{\hat{\tau}}$, that these two quotient spaces can be identified in a canonical way.

3.3. Proof of Theorem 2. If we inductively repeat the above surgery procedure along the minimal stratum, we will eventually obtain a symplectic manifold $\widetilde{W}$ with a free Hamiltonian $\mathbb{S}^{1}$-action $\widetilde{\beta}$. The symplectic reduced space of this action at the level 0 will be a smooth symplectic manifold $\widetilde{M}$ and it will be equipped with a function $f: \widetilde{M} \rightarrow M$, which factors through all the previous steps of the resolution (that is, reduced spaces with orbifold singularities of decreasing order), and is a symplectic diffeomorphism outside an arbitrarily small neighbourhood of the set of orbifold singularities of $M$.

\section{Applications}

4.1. Isolated cyclic orbifold singularities. Any isolated cyclic orbifold singularity can be represented as $\mathbb{C}^{n} / \mathbb{Z}_{k}$ with symplectic form $\omega=$ $i \sum_{j=1}^{n} d z_{j} \wedge d \bar{z}_{j}$, where the generator $\xi=e^{2 \pi i / k}$ of $\mathbb{Z}_{k}$ acts by

$$
\xi \cdot \mathbf{z}=\left(e^{2 \pi i / k} z_{1}, e^{2 \pi i a_{2} / k} z_{2}, \ldots, e^{2 \pi i a_{n} / k} z_{n}\right),
$$

and $a_{2}, \ldots, a_{n} \in \mathbb{N}$ are all coprime with $k$.

To find a resolution of the singularity using Theorem 2.3, just note that $\mathbb{C}^{n} / \mathbb{Z}_{k}$ can be obtained by doing symplectic reduction on the manifold $\mathbb{C}^{*} \times$ $\mathbb{C}^{n}$ with $\mathbb{S}^{1}$-action given by

$$
e^{i \varphi} \cdot\left(\rho e^{i \vartheta}, z_{1}, \ldots, z_{n}\right):=\left(\rho e^{i(k \varphi+\vartheta)}, e^{i \varphi} z_{1}, e^{i a_{2} \varphi} z_{2}, \ldots, e^{i a_{n} \varphi} z_{n}\right),
$$

symplectic form $\omega+\rho d \rho \wedge d \vartheta+\frac{1}{k} d \vartheta \wedge \sum_{j=1}^{n} a_{j} d\left|z_{j}\right|^{2}$ and moment map $H\left(\rho e^{i \vartheta}, z_{1}, \ldots, z_{n}\right)=\rho^{2}$. Theorem 2.3 then immediately implies the following generalisation of the desingularisation result obtained in dimension 4 :

Corollary 4.1. Every symplectic orbifold with only isolated cyclic singularities admits a symplectic resolution.

Cavalcanti et al. [CFM08] recently introduced a method that allows one to find resolutions for all isolated symplectic orbifold singularities.

\subsection{Generalized Boothby-Wang fibrations are fillable.}

Definition. A Boothby-Wang fibration is a closed contact manifold $(P, \alpha)$ with a free $\mathbb{S}^{1}$-action which is given by the flow of the Reeb field $X_{\text {Reeb }}$. A generalized Boothby-Wang fibration is a closed contact manifold $(P, \alpha)$, where the Reeb field induces a locally free $\mathbb{S}^{1}$-action. 
Remark 4.2. Any closed contact manifold $(P, \alpha)$ with a (locally) free $\mathbb{S}^{1}$ action that leaves the contact structure $\xi=\operatorname{ker} \alpha$ invariant, and is transverse to it, gives rise to a (generalised) Boothby-Wang fibration: Just average the contact form $\alpha$ over the circle action. Then we have that $F:=\alpha\left(X_{P}\right)$ is a non zero $\mathbb{S}^{1}$-invariant function. Define $\alpha^{\prime}:=\frac{1}{F} \alpha$. This gives a new $\mathbb{S}^{1}$-invariant contact form, for which we have $\alpha^{\prime}\left(X_{P}\right)=1$. Furthermore, $d \alpha^{\prime}\left(X_{P},-\right)=\mathcal{L}_{X_{P}} \alpha^{\prime}-d\left(\alpha^{\prime}\left(X_{P}\right)\right)=0$, so that $X_{P}$ is the Reeb field for $\alpha^{\prime}$.

Remark 4.3. A Boothby-Wang fibration $(P, \alpha)$ defines an $\mathbb{S}^{1}$-principal bundle over the manifold $B=P / \mathbb{S}^{1}$ with connection 1 -form $\alpha$. The curvature form $\omega$ is the unique 2 -form on $B$ that satisfies $\pi^{*} \omega=d \alpha$. The base manifold $(B, \omega)$ is a symplectic manifold and $\omega$ represents an integral cohomology class.

Conversely, for any symplectic manifold $(B, \omega)$ with integral symplectic form, one can construct a Boothby-Wang fibration $(P, \alpha)$ over it, the socalled pre-quantization. This is the inverse of the previous construction.

A generalized Boothby-Wang fibration can be considered as the prequantization of the symplectic orbifold $\left(P / \mathbb{S}^{1}, \omega\right)$, and all the statements made in Remark 4.3 can be translated to this setting.

Proposition 4.4. A generalized Boothby-Wang fibration $(P, \alpha)$ has a natural convex filling by a symplectic orbifold.

Proof. These computations were obtained with the help of H. Geiges. Consider the (complex) "line bundle" $L$ associated to $P$, i.e., the bundle obtained from $P \times \mathbb{C}$ by identifying $(p, z)$ with $\left(e^{-i \varphi} * p, e^{i \varphi} z\right)$ for every $e^{i \varphi} \in \mathbb{S}^{1}$. The manifold $P$ embeds naturally via

$$
P \hookrightarrow L, \quad p \mapsto[p, 1] .
$$

Define on $P \times \mathbb{C}$ the 1 -form

$$
\gamma:=\frac{1}{2}\left(\left(1+|z|^{2}\right) \alpha+x d y-y d x\right) .
$$

It is easy to check that $\gamma$ is $\mathbb{S}^{1}$-invariant, and that $\gamma\left(X_{P \times \mathbb{C}}\right) \equiv-1 / 2$ for the generator of the circle action $X_{P \times \mathbb{C}}=-X_{P}+x \partial_{y}-y \partial_{x}$ (recall that $X_{P}$, the generator of the circle action on $P$, coincides with the Reeb field $X_{\text {Reeb }}$ of $\alpha$ ). Its differential

$$
\omega:=\frac{1}{2} d\left(|z|^{2}\right) \wedge \alpha+d x \wedge d y+\frac{1+|z|^{2}}{2} d \alpha
$$

defines hence a well-defined 2-form on $L$ that is even symplectic, because $2^{n} \omega^{n}=n\left(1+|z|^{2}\right)^{n-1}(d \alpha)^{n-1} \wedge\left(d|z|^{2} \wedge \alpha+2 d x \wedge d y\right)$ has only a onedimensional kernel on $P \times \mathbb{C}$ that is generated by $X_{P \times \mathbb{C}}$. It follows that $L$ is a symplectic orbifold where all orbifold singularities sit along the zero section. 
Finally, the following field

$$
X:=\frac{1+r^{2}}{2 r} \partial_{r}=\frac{1+x^{2}+y^{2}}{2\left(x^{2}+y^{2}\right)}\left(x \partial_{x}+y \partial_{y}\right)
$$

is a Liouville vector field for the manifold $(P, \alpha)$, and $(L, \omega)$ is hence a convex filling of $P$.

Another, more complicated, way to obtain the symplectic orbifold $L$ is as the symplectic reduction of the Hamiltonian $\mathbb{S}^{1}$-manifold described below. Since the orbifold singularities lie in the interior of $L$, by passing then to a symplectic resolution of $L$ whose existence is guaranteed by Theorem 2.3, we can obtain a smooth symplectic filling of the contact manifold $(P, \alpha)$.

The Hamiltonian $\mathbb{S}^{1}$-manifold $(-\varepsilon, \varepsilon) \times P \times \mathbb{C}=\{(t, p, z)\}$ with the circle action induced from $P \times \mathbb{C}$, and symplectic form

$$
\Omega:=d((1+t) \gamma)
$$

has Hamiltonian function $H(t, p, z)=t / 2$. Hence the symplectic reduction at 0 gives back the symplectic orbifold $L$ defined above.

Corollary 4.5. Generalized Boothby-Wang fibrations are symplectically fillable by a smooth manifold.

Remark 4.6. (1) Popescu-Pampu recently proved the following conjecture of Biran: there exist pre-quantizations (e.g., on higher dimensional tori) that are not holomorphically fillable [PP08]. These manifolds, on the other hand, do have a strong symplectic filling, showing that the different types of fillability do not coincide (a result well-known in dimension 3).

(2) Massot showed that any contact structure on a three-dimensional Seifert manifold that is transverse to the fibres (but not necessarily invariant) has a weak symplectic filling [Mas08]. To achieve this result he constructs first a filling by an orbifold, whose singularities can then be resolved by using for example the method presented in this paper.

\section{References}

[CFM08] G. Cavalcanti, M. Fernández and V. Muñoz, Symplectic resolutions, Lefschetz property and formality, Adv. Math. 218(2) (2008), 576-599.

[GGK99] V. Ginzburg, V. Guillemin and Y. Karshon, The relation between compact and non-compact equivariant cobordisms, Tel Aviv Topology Conference: Rothenberg Festschrift (1998), Contemp. Math., Vol. 231, Amer. Math. Soc., Providence, RI, 1999, 99-112.

[God01] L. Godinho, Blowing up symplectic orbifolds, Ann. Global Anal. Geom. 20(2) (2001), 117-162. 
[Kir85] F. Kirwan, Partial desingularisations of quotients of nonsingular varieties and their Betti numbers, Ann. of Math. (2) 122(1) (1985), 41-85.

[Ler95] E. Lerman, Symplectic cuts, Math. Res. Lett. 2(3) (1995), 247-258.

[Mas08] P. Massot, Geodesible contact structures on 3-manifolds, Geom. Topol. 12(3) (2008), 1729-1776.

[MS98] D. McDuff and D. Salamon, Introduction to symplectic topology. 2nd ed., Oxford Mathematical Monographs, Oxford University Press, New York, NY, 1998.

[MS99] E. Meinrenken and R. Sjamaar, Singular reduction and quantization, Topology 38(4) (1999), 699-762.

[PP08] P. Popescu-Pampu, On the cohomology rings of holomorphically fillable manifolds, Singularities II: Geometric and Topological Aspects, Vol. 475, 2008, 169188.

[Rei] M. Reid, Cyclic surface quotient singularities, http://www.maths.warwick.ac. uk/ miles/surf/more/cyclic.pdf.

[Wei77] A. Weinstein, Symplectic V-manifolds, periodic orbits of Hamiltonian systems, and the volume of certain Riemannian manifolds, Comm. Pure Appl. Math. 30(2) (1977), 265-271.

ÉCOle Normale Supérieure de Lyon

Unité de Mathématiques Pures et Appliquées

UMR CNRS 5669

46, ALLÉE D'ITALIE

69364 LYON CEDEX 07

FRANCE

E-mail address: kniederk@umpa.ens-lyon.fr

Department of Mathematics, Faculty of Sciences

VRIJE UNIVERSITEIT

De BoelelaAN 1081A

1081 HV AMSTERDAM

THE NeTHERLANDS

E-mail address: pasquott@few.vu.nl

Received 12/10/2007, accepted 02/19/2009

Klaus Niederkrüger is working at the ENS de Lyon, where he is being funded by the Agence Nationale de la Recherche (ANR) project Symplexe BLAN 06-3-137237. Federica Pasquotto is working at the Vrije Universiteit Amsterdam and is supported by VENI Grant 639.031.620 of the Nederlandse Organisatie voor Wetenschappelijk Onderzoek (NWO).

We thank the Mathematisches Forschungsinstitut Oberwolfach for inviting us to a Research in Pairs stay at the beginning of 2007, during which we developed the main parts of this article. We also thank the referee for reading our manuscript very carefully and for many useful remarks and suggestions. 
